\title{
Conhecimento e atividades da enfermagem no cuidado do esquizofrênico ${ }^{1}$
}

\author{
Nurses' knowledge and expectations regarding the schizofrenic care \\ Conocimiento y expectativas de los enfermeros en el cuidado de lo esquizofrenico
}

\author{
Sueli Aparecida de Castro', Antonia Regina Ferreira Furegato"
}

\section{RESUMO}

Em sua prática, os enfermeiros enfrentam dificuldades na implementação do cuidado ao esquizofrênico. O objetivo deste trabalho foi identificar conhecimento e atividades da enfermagem no cuidado esquizofrênico. 0 presente estudo realizado em 2004 é do tipo exploratório-descritivo, com análise qualiquantitativa. Participaram da entrevista 17 dos 20 enfermeiros de um hospital público, 8 fizeram especialização em Enfermagem Psiquiátrica e a maioria prefere trabalhar com doentes mentais crônicos. Os enfermeiros e a equipe de enfermagem referem dificuldades no manejo dos pacientes esquizofrênicos com sintomas de heteroagressividade, delírios, alucinações e imprevisibilidade. Há poucas referências a respeito do aumento da autonomia do paciente e da participação da família no procedimento terapêutico. A maior dificuldade dos enfermeiros reside na comunicação e nas relações interpessoais. Concluindo, os enfermeiros cuidam dos pacientes de acordo com a sintomatologia, demonstram preocupação com a assistência prestada e relatam a necessidade de buscar maior conhecimento.

Palavras chave: Enfermagem psiquiátrica; Esquizofrenia; Conhecimento; Comunicação.

\section{ABSTRACT}

Nurses face difficulties in their practice regarding the implementation of care to the schizophrenic. This article aims to identify the knowledge and activities of the nurse's team about the schizophrenic care. This study realized in 2004 is the type exploratorydescriptive, with quality-quantitative analysis. Seventeen of 20 nurses of a public hospital participated in the interview, eight specialized in Psychiatric Nursing and the majority prefers to work with chronic patients. Nurses and the nursing team report difficulties in the management of schizophrenic patients with symptoms of heteroagressiveness, delirium, hallucinations and imprevisibility. There are few references regarding the patient's autonomy
}

and the family participation in the therapeutic procedure. The nurses' greater difficulty is on communication and interpersonal relations. Concluding, nurses assist the patients according to syntomatology, show concern with the assistance delivered and report the need to seek knowledge.

Key words: Psychiatric nursing; Schizophrenia; Knowledge; Communication.

\section{RESUMEN}

En su práctica, los enfermeros enfrentan dificultades en la implementación del cuidado al esquizofrénico. El objetivo de este trabajo es identificar conocimiento y actividades de la enfermería, cuanto al cuidado del esquizofrenico. Este estudio en el año 2004 es el tipo exploratorio-descriptivo, con la calidad de análisis cualitativo y cuantitativo. Participaron de la entrevista 17 de los 20 enfermeros de un hospital publico, 8 hicieron especialización en Enfermería Psiquiátrica y la mayoría prefiere trabajar con enfermos mentales crónicos. Los enfermeros y el equipo de enfermería, hacen referencia a las dificultades en el manejo de los pacientes esquizofrénicos con síntomas de heteroagresividad, delirios, alucinaciones e imprevisibilidad. Hay pocas referencias al respecto de mayor autonomía del paciente e participación de la familia en el procedimiento terapéutico. La mayor dificultad de los enfermeros reside en la comunicación y en las relaciones interpersonales. Concluyendo, los enfermeros cuidan de los pacientes de acuerdo con la sintomatología, demuestran preocupación con la asistencia y relatan la necesidad de buscar mayor conocimiento.

\footnotetext{
${ }^{1}$ Parte do TCC no Curso de Especialização em Enfermagem Psiquiátrica e Saúde Mental EER/USP.

Enfermeira do Hospital Santa Tereza de Ribeirão Preto, mestranda em Enfermagem Psiquiátrica. E-mail: castrossueli@hotmail.com

"Prof. Titular do Depto de Enfermagem Psiquiátrica Ciências
} Humanas da EERP/USP. E-mail: furegato@eerp.usp.br 


\section{Palabras clave: Enfermería psiquiátrica;}

\section{NTRODUÇÃO}

O termo esquizofrenia foi criado no inicio do século passado por um psiquiatra suíço, Eugen Bleuler que se destacou no universo psiquiátrico; considerava esse diagnóstico clinico como uma das doenças mentais mais severas $^{(1)}$.

Emil Kraepelin denominou a esquizofrenia de Demência Precoce. Esses critérios baseavam-se em três características principais: sintomatologia, etiologia e evolução. O critério sintomatológico constituía-se, na descrição dos sintomas observados, tais como déficits da atenção e da compreensão, as alucinações, principalmente auditivas, o pensamento sonoro, a vivência de influência, o embotamento da afetividade e as mudanças do comportamento. O critério etiológico pressupunha a existência de um quadro endógeno. $O$ critério evolutivo caracterizava-se pela evolução desfavorável e pela invalidez psíquica. Daí a denominação de Demência Precoce ${ }^{(1)}$.

A visão pessimista de Kraepelin, quase centenária, tem persistido no moderno sistema de diagnóstico. Por isso, não é surpresa que muitos clínicos, pacientes e familiares conformem-se que indivíduos esquizofrênicos sejam desabilitados, com pequena expectativa para desenvolver atividades produtivas na sociedade ${ }^{(2)}$.

Esta visão causa repercussão na qualidade da assistência dispensada ao esquizofrênico, estigmatizando-o, reduzindo seu tratamento à exclusão manicomial. Nesta perspectiva, os profissionais da saúde limitam seu tempo e habilidades à contenção e aos mínimos cuidados de higiene e sobrevivência.

A família era vista como propiciadora do adoecimento, ratificando a necessidade da retirada da pessoa doente deste meio(3). Assim, a família não se constituía como parte do tratamento. A sociedade se encarregava de manter a doença e conter o doente dentro dos seus muros.

A esquizofrenia pode ser definida através de exames clínicos, levando-se em conta o contexto social em que o processo psiquiátrico está sendo conduzido. É caracterizada por sintomas psicóticos (distúrbios do pensamento, alucinações e delírios), além de alterações do
Esquizofrenia; Conocimiento; Comunicación.

desempenho social e pessoal do paciente. É uma doença da personalidade que afeta a zona central do "eu", alterando a estrutura vivencial, interferindo na capacidade de vida e de convivência da pessoa com a realidade ${ }^{(1,4)}$.

$O$ refinamento do conhecimento sobre o curso clínico da esquizofrenia, o melhoramento das técnicas psicoterápicas, a introdução de novos medicamentos antipsicóticos e o aprimoramento da condução terapêutica vem alterando a visão da pessoa afetada. O conjunto dessas ações pode alterar a avaliação do curso da doença aumentando suas chances terapêuticas. Isso tudo pode evitar cronificações institucionais, através de estratégias reabilitadoras e de reinserção social.

As estratégias de detecção e intervenção precoce nas psicoses seguem o modelo de outras áreas da medicina em busca da prevenção seletiva (antes do inicio da doença) e da prevenção indicada (logo no nas primeiras manifestações de suspeita de doença, antes de preencher critérios para diagnóstico ${ }^{(5)}$.

A mudança de perspectiva sobre a esquizofrenia (não mais como uma doença maligna e deteriorante) bem como os efeitos da reforma psiquiátrica (saindo do modelo asilar para o tratamento na comunidade - NAPS, CAPS, Ambulatórios de Saúde Mental, Hospital Dia, etc.) tornaram estes pacientes visíveis aos profissionais e à sociedade.

Em conseqüência, as formas de exercer a psiquiatria, através da reclusão, vêm sendo repensadas e questionadas. O profissional que presta assistência ao esquizofrênico deve estabelecer relações com propósito determinado e adequado às necessidades culminando em projetos terapêuticos, estabelecidos pela equipe de profissionais e com participação ativa do portador e seu familiar. Essa relação se confirma na medida em que se estabelecem vínculos de empatia e aliança.

A enfermeira, através do cuidado nas 24 horas deve partir do princípio de que o portador dessa patologia é um ser humano singular que apresenta alterações emocionais e comportamentais. Assim, poderá ajudá-lo a enfrentar as dificuldades, aceitando suas limitações. Os profissionais devem acreditar na remissão dos sintomas e na capacidade de 
inserção social dessa pessoa, apesar da doença. O enfermeiro e sua equipe precisam dirigir suas ações para atender as necessidades apresentadas pelo esquizofrênico.

Os enfermeiros, juntamente com os demais membros da equipe, desempenham papel fundamental no cuidado e na luta contra o estigma da esquizofrenia, em todas as fases do tratamento e da recuperação das pessoas afetadas.

$\mathrm{Na}$ prática, observa-se que enfermeiros psiquiátricos, com freqüência, encontram dificuldades em implementar os cuidados de enfermagem aos esquizofrênicos, devido as características dessa doença mental. As manifestações tipo alucinações, delírios, autoagressão e agitação psicomotora provocam ansiedade nos enfermeiros, dificultando o estabelecimento da comunicação interpessoal e a concretização da relação de ajuda.

Como profissionais envolvidos com o cuidado ao esquizofrênico e com o ensino de enfermagem psiquiátrica temos várias questões que precisam ser melhor compreendidas incluindo-se a relação enfermeiro - paciente na perspectiva daqueles que cuidam diariamente.

O objetivo deste estudo foi, portanto, identificar conhecimento e atividades da enfermagem no cuidado ao portador de esquizofrenia.

\section{METODOLOGI A}

O presente estudo é do tipo exploratóriodescritivo, com análise quali-quantitativa. A coleta dos dados realizou-se no Hospital Psiquiátrico de Ribeirão Preto. No ano de 2004, o hospital contava com 280 leitos, distribuídos nos setores de agudos masculino e feminino, crônicos, dependentes químicos, geriatria masculina e feminina, clínica médica e reabilitação. É uma unidade de internação que vem paulatinamente agregando os conceitos da reforma.

O critério para inclusão dos sujeitos na pesquisa era ser profissional pertencente ao quadro de enfermeiros do hospital. No momento da coleta dos dados havia 20 enfermeiros trabalhando, dos quais 17 aceitaram participar do estudo.

O instrumento de coleta dos dados foi um questionário semi-estruturado composto por duas partes: a primeira continha questões para identificação do sujeito (idade, sexo, estado conjugal e experiência profissional); a segunda constava de um roteiro com questões abertas para identificar o conhecimento sobre esquizofrenia, suas preferências e dificuldades no trabalho, bem como suas expectativas.

O projeto foi aprovado pelo Comitê de Ética da EERP-USP (no 475/2004) com consentimento da Instituição onde os dados foram coletados. O instrumento foi entregue aos sujeitos que, após os esclarecimentos, assinaram o Termo de Consentimento e responderam ao questionário.

Para a análise, procedeu-se à caracterização dos sujeitos e suas respostas foram avaliadas por agrupamento de todos os elementos com características de concordância e discordância. Os dados foram discutidos com base na literatura sobre o tema e com considerações possíveis a partir do olhar do pesquisador.

\section{RESULTADOS E DI SCUSSÃO}

\section{Caracterização dos sujeitos e sua relação com o trabalho}

O estudo foi realizado em um hospital psiquiátrico na cidade de Ribeirão Preto (SP). Foram entrevistados 17 enfermeiros (17:20), cuja idade variou de 28 a 50 anos. Um terço dos enfermeiros (7:17) está na faixa dos 40 aos 50 anos. São 14 do sexo feminino (82\%). Formaram-se entre as décadas de 80 e 90 (16:17).

O tempo de trabalho dos enfermeiros na psiquiatria varia de 4 a 18 anos. Dez enfermeiros sentem-se realizados na profissão (59\%). A maioria dos satisfeitos trabalha somente na enfermagem psiquiátrica.

Muitos dos que disseram não para a realização profissional preferem trabalhar com pacientes na fase aguda da doença (UE, Agudos) e 6 dos 17 sujeitos preferem trabalhar com pacientes crônicos,

A enfermeira e a equipe de enfermagem estão em contato mais próximo e mais prolongado com os doentes, resultando em maior desgaste e muitas vezes em mais stress e insatisfação ${ }^{(6-7)}$.

Houve marcante rejeição (12:17) por Unidade de Internação em Hospital Geral. Esse resultado pode refletir a dificuldade dos 
enfermeiros em trabalhar nessa nova proposta, que inclui a construção de novos modelos de assistência, inclusive nas enfermarias de psiquiatria em hospital geral. É importante pensar que o espaço de inclusão do doente mental exige que os profissionais construam novas práticas, mais éticas e mais flexíveis, beneficiando a pessoa em sofrimento. É uma psiquiatria integrada com a comunidade, preocupada em tratar o doente, cuidar do seu sofrimento e aumentar sua autonomia.

Talvez a dificuldade apresentada pelos enfermeiros seja conseqüência da maneira de trabalhar em uma instituição psiquiátrica tradicional, na qual se desenvolvem ações voltadas para o tratamento das alterações psicopatológicas, referencial marcadamente biológico.

Os enfermeiros relatam que preferem trabalhar com pacientes crônicos. Essa preferência pode refletir a satisfação pelos resultados adquiridos com o trabalho de ressocialização, através do qual se consegue, em muitos casos, devolver à sociedade pessoas que por muito tempo foram excluídas. Ao contrario, os pacientes, na fase aguda da doença despertam nos profissionais, sentimentos de impotência pelas demandas sintomáticas e pela incerteza das perspectivas. Um estudo que identificou a satisfação de profissionais da saúde mental (enfermeiros) ao contribuírem com a ressocializacao dos pacientes evidenciou que o trabalho é muito difícil porque exige tempo e recursos, mas os resultados positivos são gratificantes ${ }^{(8)}$.

Por outro lado, os pacientes com doença crônica não são muito questionadores ou exigentes, causando menos desgaste para a equipe de enfermagem. Quem trabalha com pacientes na fase aguda da doença precisa ser apto a lidar com sua sintomatologia e, também com os seus familiares aflitos e desesperados.

É necessário reconhecer que há novas exigências e mudanças significativas no trabalho dos enfermeiros, as quais não estão voltadas somente para o tratamento da patologia, mas para o resgate da subjetividade e todo o contexto de vida dessas pessoas.

A relação de ajuda com estes pacientes exige grande disponibilidade afetiva, bem como compreensão ampla de sua condição de ser no mundo, perdas e desilusões. Para estabelecer a relação de ajuda é necessário que a enfermeira tenha como base uma postura ou um modo de ação que seja coerente com seu modo de ser e de encarar a vida, caso contrario será difícil entrosar-se com a equipe e ajudar efetivamente os pacientes ${ }^{(9)}$.

A relação compreensiva pode ser descrita como uma relação de suporte em todos os níveis, pois 0 enfermeiro deve possuir competências pessoais e conhecimentos profissionais para ajudar o esquizofrênico a conquistar o seu espaço.

O objetivo principal é despertar o interesse do esquizofrênico pela vida, uma vida digna e de participação no seu meio familiar e social, apesar da doença.

Nesse contexto, cresce a importância da capacitação dos enfermeiros, voltada para essa modalidade de assistência, o que poderá causar impacto positivo na qualidade do cuidado e na remissão de sintomas.

Pelas respostas dos sujeitos dessa pesquisa, constatou-se que 17 enfermeiros fizeram algum curso de especialização, sendo que 8 se especializaram em enfermagem psiquiátrica. O fato da maioria dos enfermeiros ter feito algum curso de especialização em enfermagem psiquiátrica e trabalharem somente no hospital psiquiátrico reforça a importância da busca de conhecimento.

A segurança e a confiança adquiridas num curso de especialização podem favorecer a melhor atuação do enfermeiro, ampliação do seu comprometimento profissional e desenvolvimento de atitudes profissionais mais terapêuticas e compreensivas ${ }^{(10)}$.

\section{I dentificação do conhecimento do enfermeiro em relação ao esquizofrênico}

Os enfermeiros indicaram, em suas respostas, que têm dificuldades em prestar assistência aos pacientes heteroagressivos, delirantes e com alucinações. Indicam também que a dificuldade reside na comunicação e nas relações interpessoais.

O paciente considerado pelos enfermeiros como o mais fácil para o manejo foi o crônico. Os deficientes e os dementes são os indicados como menos complexos para se prestar assistência.

Cinco enfermeiros apontam que o paciente depressivo é o mais difícil de manejo, 
especialmente na fase aguda da doença. Quatro responderam que o paciente com transtorno de personalidade é o mais difícil de manejo, na fase aguda. Ressaltam que o comportamento paranóide, com alucinações e delírios é ameaçador e, principalmente, aqueles com risco suicida.

Em suma, consideraram que aqueles pacientes com menor contato são os mais fáceis de manejar, por apresentar menor capacidade de comunicação, mais dependência e por ser controlados pela própria doença, pelos medicamentos e pela institucionalização.

Vale ressaltar que os sintomas destacamse independente do diagnóstico, conseqüentemente $o$ enfermeiro cuida do paciente de acordo com o comportamento apresentado.

É importante valorizar e incentivar os pacientes a participarem do seu tratamento, para que as chances de adesão ao tratamento possam ser maiores. Os pacientes com pior crítica (relacionamento ruim com médico durante a internação ou experiências negativas durante a internação) apresentam uma atitude negativa em relação ao tratamento ${ }^{(11)}$.

Tal afirmação leva-nos a perceber a importância de ajudar o paciente, portador de doença crônica a se tornar o menos dependente possível, através do planejamento e implementação do cuidado que o ajude enfrentar e superar as dificuldades para alcançar uma qualidade de vida melhor. Muitas vezes, uma aparente passividade pode ser erroneamente reconhecida como uma característica inerente ao seu processo de cronicidade da doença ${ }^{(12)}$.

Por outro lado, os entrevistados não referiram nenhuma vez como se comunicam com o paciente que conhece a sua doença, suas reações, ou aquele que está orientado quanto ao tratamento, que está num nível positivo de evolução da doença e se percebe ativo neste processo. Certamente esta conduta não deve fazer parte de sua rotina de trabalho. Essa maneira de pensar está vinculada ao paradigma dominante na atenção hospitalar, o qual está focado no controle, bastando que, na internação, o paciente se comporte de acordo com as regras institucionais.

$\mathrm{Na}$ verdade os profissionais de saúde e principalmente os enfermeiros devem estimular o exercício da autonomia desses clientes. Tal conduta é um processo ético do cuidado que deve ser constantemente buscado e estimulado ${ }^{(12)}$

Como se observou neste estudo, as respostas dos enfermeiros continuam enfatizando a condição do sujeito e não o sujeito.

Os pacientes crônicos são mais "estáveis" e, portanto exigem menos tempo da equipe. $\mathrm{Na}$ verdade, eles deveriam ter a atenção da equipe tanto quanto o paciente agudo, para conseguir gerir sua própria vida. Para que os portadores de patologias crônicas posam conviver com limitações impostas por sua condição de cronicidade, é necessário que os cuidadores, em especial as enfermeiras, estimulem o processo de compreensão desses clientes no que se refere ao enfrentamento dessas limitações ${ }^{(12)}$.

Quando se questiona o tipo de paciente que requer mais atenção aqueles que apresentam depressão foram os mais citados 13:17 (76\%). Um dos aspectos mais ressaltados pelos participantes foi a preocupação com o risco suicida.

É fundamental adotar medidas de proteção que previnam ou diminuam as situações de risco ao deprimido hospitalizado buscando reverter ou impedir a ideação. A alternativa de internação pode servir como uma defesa, como uma atitude protetora. A adesão à medicação antidepressiva é essencial para consolidação da resposta ao tratamento, prevenção de recaídas e reincidência ${ }^{(13-14)}$.

A adesão ao tratamento é processo multifatorial que se estrutura em uma parceria entre quem cuida e quem é cuidado; diz respeito, à freqüência, à Constancia eà perseverança na relação com o cuidado em busca da saúde. Portanto, o vinculo entre profissionais e paciente é fator estruturante e de consolidação do processo, razão pela qual deve ser cosistente para que se efetive ${ }^{(15)}$.

Entende-se que todos os profissionais da saúde devem questionar se suas ações conseguem efetivamente contribuir, não apenas para a manutenção da integridade física, mas para a diminuição dos agravos à saúde e para melhor qualidade de vida dos pacientes em seu cotidiano.

Neste estudo, a grande maioria dos enfermeiros (16:17) acredita na cura da 
depressão, na cura da ansiedade (15:17) e, quase todos, acreditam na cura do uso de drogas (16:17) e do álcool (17:17). Entretanto, a esquizofrenia, juntamente com transtorno bipolar e o transtorno de personalidade são, na opinião dos enfermeiros, as patologias com pior probabilidade de cura (13:17)

Sete enfermeiros indicam que têm dificuldades com pacientes heteroagressivos, quatro com pacientes delirantes e quatro com pacientes que apresentam alucinações. Indicam também que a dificuldade reside na comunicação, nas relações interpessoais, com possibilidade de reversão da dificuldade após controle químico da sintomatologia negativa.

Chama a atenção que os enfermeiros não se referem a certos sintomas muito freqüentes na esquizofrenia como é o caso do alheamento, a falta de interesse pelas pessoas, por si mesmo ou pelas coisas que comumente as pessoas se interessam. Estes sintomas não "incomodam" diretamente a equipe e, portanto, são negligenciados.

Os enfermeiros admitem que tais dificuldades se estendem aos auxiliares de enfermagem. Este fato reafirma as próprias dificuldades do enfermeiro, as quais acabam por refletir nas ações dos auxiliares, que são coordenados, supervisionados e orientados pelo enfermeiro. Quando se refere ao que o outro pensa, sente ou faz se está colocando nestas figuras o que se pensa e sente a respeito do objeto focalizado.

Os enfermeiros sabem que os auxiliares são importantes elementos da equipe de enfermagem. Entretanto, nem sempre estão devidamente preparados para abordar o paciente, para reconhecer as manifestações da doença, assim como lhes falta conhecimento para entender o comportamento ou faltam informações técnicas para executar estas tarefas. Os enfermeiros desta pesquisa não se referem à sua responsabilidade no preparo, orientação e supervisão destes auxiliares.

Pode ser que os enfermeiros não abordem os auxiliares sobre estas questões porque eles próprios não se sintam preparados e prontos para capacitá-los. Costumam justificar por "falta de tempo" ou sobrecarga de trabalho. Os que reconhecem esta carência buscam cursos de especialização, atualização e aperfeiçoamento.

Pesquisa para conhecer as convicções e as preocupações de enfermeiros psiquiátricos sobre "ataques" de pacientes e segurança no ambiente de trabalho verificou que a maioria dos profissionais pensava que os membros da equipe que trabalham com pacientes mentalmente enfermos podem ser fisicamente agredidos em algum momento de sua carreira profissional $^{(16)}$.

O paciente que causa medo na equipe de enfermagem é aquele que apresenta risco suicida (6), o heteroagressivo (5), o usuário de drogas (3), o paciente em surto psicótico (3), segundo as respostas dos sujeitos desta pesquisa.

Causam medo nos enfermeiros os comportamentos que denunciam a falta de cuidado, a negligência com seu trabalho de vigiar e sua impotência para dominar a situação (fuga, suicídio, mudança de comportamento, agressão, comportamento auto e hetero destrutivos). Isso assusta o enfermeiro que, ainda hoje, carrega o fardo do controle, da vigilância e da garantia da execução do trabalho penoso.

Os resultados do presente estudo indicam que os enfermeiros cuidam de acordo com o comportamento apresentado, agindo de forma consciente, porém individualizada e sugerindo que não existe padronização de condutas para nortear a tomada de decisões com vistas a melhor realização do seu trabalho.

Quando indagados sobre sintomas de alheamento e isolamento, a maioria dos enfermeiros (14:17) tenta a comunicação pessoal para ajudar o paciente a sair da situação e os demais apenas estimulam a participação em alguma atividade.

A comunicação permite ao enfermeiro aproximar-se das pessoas ao seu redor, no exercício de suas atividades profissionais. Entretanto, o enfermeiro muitas vezes comunica-se com o paciente sem que isto se reverta em beneficio mútuo e sem que ele próprio sinta-se satisfeito ou seguro das ações que efetuou ${ }^{(9,17)}$.

O enfermeiro sabe que precisa comunicarse conscientemente oferecendo dados da realidade e conduzir o doente para atividades e para o convívio social, evitando o isolamento. Sabe que deve orientar a equipe de enfermagem. Entretanto percebe-se que 0 enfermeiro encontra dificuldades para executar, 
na prática do seu trabalho, as técnicas do relacionamento interpessoal e orientar a equipe de enfermagem. Alega falta de tempo ou excesso de atividade burocrática ${ }^{(9)}$.

Apenas 12 dos 17 enfermeiros preparam o paciente internado para a alta, orientando sobre medicações e retorno. Mesmo, assim, a maioria só o faz na "saída", quando o paciente está de alta e passa rapidamente pela orientação de enfermagem. Apenas quatro enfermeiros mencionam a família, poucos se referem às atividades associadas a outras orientações e à vida social do paciente.

A alta deve ser preocupação da equipe desde o momento da entrada do paciente no serviço psiquiátrico, para que futuramente ele seja co-participante no seu tratamento. Estudos têm mostrado que o ideal é que o planejamento de alta seja iniciado logo após a admissão do cliente ou mesmo antes da internação, em nível ambulatorial, com a identificação das suas necessidades reais ou potenciais ${ }^{(12)}$.

A maioria dos enfermeiros (13) respondeu que os pacientes esquizofrênicos têm boas perspectivas desde que façam tratamento medicamentoso; sete enfermeiros responderam que esquizofrênicos têm boas condições aderindo ao tratamento e tendo apoio familiar.

O tratamento e o apoio familiar são, de fato, dois pontos de suma importância. Se a pessoa ficar isolada na sua casa só tomando medicamentos, a perspectiva não é melhor do que num asilo, pois ele fica cultivando apenas a doença e pouco aprende a desenvolver suas potencialidades para restringir $o$ grau de dependência. Os enfermeiros lembram que $o$ esquizofrênico sempre volta. Assim surgem algumas questões:

- ele está recebendo tratamento adequado com a orientação médica e de enfermagem e dos demais elementos da equipe de profissionais?

- a equipe de enfermagem sabe identificar e manejar todos os sintomas próprios da esquizofrenia?

- ele está tendo acompanhamento, observando-se as manifestações da doença e os efeitos das terapias medicamentosas, psicológicas e sociais?

- os serviços de acompanhamento estão estimulando o paciente e a família a entenderem a doença, a participarem do tratamento medicamentoso, a buscarem outros recursos terapêuticos (psicologia, acompanhamento terapêutico, terapia ocupacional, recreacional) e a se inserirem na comunidade (grupos de auto-ajuda, escola, trabalho remunerado ou voluntário, atividades religiosas, atividades esportivas ou culturais)?

Estes questionamentos são importantes para compreender a reforma psiquiátrica de forma contextualizada observando-se vários aspectos relacionados às peculiaridades econômicas, políticas, culturais e sociais do país ${ }^{(18)}$. Ainda é importante articular esses fatores com a finalidade de estabelecer estratégias para lidar com a realidade através de intervenções mais assertivas para facilitar a adesão ao tratamento, a reinserção social, e conseqüentemente repercussão na redução das reinternações psiquiátricas, principalmente para as doenças crônicas, como no caso a esquizofrenia.

Para o esquizofrênico, existe ainda um grande estigma vinculado a uma visão socialmente negativa, que dificulta uma real inserção do paciente em sua comunidade ${ }^{(19)}$.

A esquizofrenia $e$ as outras doenças mentais, em geral, ainda são tratadas com preconceito e os enfermeiros, juntamente com os demais profissionais das equipes de saúde devem ser agentes desmistificadores para romper com seus próprios mitos e preconceitos em relação à doença, transformando sua maneira de cuidar.

A partir do momento em que o paciente se sente valorizado e integrado em seu processo de cuidar, ele pode sentir que também é responsável por si mesmo e pelo seu próprio cuidado e conseqüentemente resgatar sua cidadania, potencialmente presente, antes de sua condição de cronicidade ${ }^{(11)}$.

A participação da família é fundamental para ajudar o paciente a ter melhores condições de vida, de tal forma que o funcionamento pessoal e social do individuo seja o melhor possível, apesar da doença.

O hospital não deve ser apenas o local de remissão dos sintomas, mas, um serviço que confira conhecimento e aptidões aos pacientes e seus familiares para manter a melhora clínica, aumentando sua autoconfiança para levar uma vida o mais próxima possível do normal, dentro 
de suas limitações ${ }^{(20)}$.

O refinamento do conhecimento sobre o curso clínico da esquizofrenia, o melhoramento das técnicas psicoterápicas, a introdução de novos medicamentos antipsicóticos e o aprimoramento da condução terapêutica bem como o oferecimento de serviços de acompanhamento e reinserção social vem alterando a visão da pessoa afetada. O conjunto dessas ações pode alterar a avaliação do curso da doença aumentando suas chances terapêuticas.

Os enfermeiros, juntamente com os demais membros da equipe, desempenham papel fundamental na luta contra o estigma da esquizofrenia, em todas as fases do tratamento e da recuperação das pessoas afetadas.

\section{CONCLUSÕES}

Quanto à caracterização dos profissionais detectou-se que a maioria está na faixa etária de 28 a 50 anos, trabalham na psiquiatria de 4 a 18 anos, a maioria freqüentou algum curso de especialização e $59 \%$ estão realizados com a profissão.

Os enfermeiros relatam que preferem trabalhar com pacientes crônicos. Essa preferência pode refletir a satisfação pelos resultados adquiridos com o trabalho de ressocialização, menor desgaste na equipe ou demonstrar as dificuldades encontradas pela enfermagem em trabalhar com os pacientes na fase aguda da doença, com sua sintomatologia e, também com os seus familiares.

Quanto ao manejo, os pacientes com menor contato, menor capacidade de comunicação, maior dependência e controlados pela própria doença são considerados os mais fáceis de manejo e aqueles com comportamentos adversos, que não apresentam comportamento submisso são desgastantes e de difícil manejo.

Os enfermeiros apresentam dificuldades em cuidar do paciente esquizofrênico devido à complexidade do transtorno por ele apresentado, como comportamentos de heteroagressividade, delírios e alucinações, principalmente no que se refere à comunicação e relações interpessoais.

A maioria acredita que esses pacientes têm boas expectativas desde que aderindo ao tratamento e tendo suporte familiar. Porém, esses profissionais, não se referem às orientações que eles próprios podem fazer para ajudar o paciente a identificar e a manejar os sintomas para estimular o auto-cuidado, diminuir o número e a gravidade das recaídas, além de orientar sobre as atividades que os serviços da rede dispõem para fazer projeções para o futuro baseado no presente e a ter uma vida mais digna e responsável.

Notou-se que os enfermeiros, ainda hoje, preocupam-se com a questão do controle. Todo comportamento do paciente que denuncie a falta de cuidado, a negligência do enfermeiro com o seu trabalho de vigiar e sua impotência para dominar situações como, fuga, suicídio, mudança de comportamento, auto e heteroagressão, assustam o enfermeiro que continua carregando o fardo do controle, da vigilância e da garantia da execução do cuidado.

Paralelamente a todas às dificuldades apresentadas, os enfermeiros acreditam que precisam observar o comportamento do paciente, que precisam comunicar-se conscientemente e que devem conduzi-lo às atividades de convívio social. Entretanto, percebe-se na prática do seu trabalho que eles ainda encontram dificuldades para implementar as técnicas de relacionamento interpessoal e para orientar a sua equipe devido ao excesso de atividade burocrática e inabilidade técnica para implementar o relacionamento interpessoal. Essa deficiência tem reflexos na evolução e na conduta de toda equipe.

A maioria dos enfermeiros diz que prepara o paciente para a alta, entretanto a maioria só o faz no momento da saída. Este trabalho deve ser desenvolvido durante todo o período da internação, pois conscientizar o paciente de que ele é um elemento ativo no seu tratamento não se faz na despedida da internação.

Diante dessas dificuldades, a equipe de enfermagem tem necessidade de melhor capacitação para o aprimoramento de conhecimentos, atualização e treinamentos para si e, conseqüentemente, para os auxiliares de enfermagem visando novas formas de abordagem, considerando as particularidades e necessidades de cada pessoa que necessita do cuidado da equipe de enfermagem.

\section{REFERÊNCI AS}

1. Shirakawa IAC, Mari JJ. O desafio da 
esquizofrenia. São Paulo: Lemos; 2001.

2. Liberman RP, Kopelowicz A, Ventura J \& Gutkind D. Operational criteria and factors related to recovery from schizophrenia. Int Rev Psychiatry. 2002; 14(4): 256-72.

3. Melman J. Família e doença mental repensando a relação entre profissionais de saúde e famílias. São Paulo: Escrituras; 2002.

4. Psiqweb [Internet]. Campinas: PsiqWeb (BR) [cited 2008 dec 31]. Psicoses. Available from: http://www.psiqweb. med.br/site/?area $=\mathrm{NO} /$ List aNoticiaBusca\&palavra =psicoses\&tipo=1\&idCate goriaNoticia=0\&pagina $=1$.

5. Louzã MR. Detecção precoce: é possível prevenir a esquizofrenia? Rev. psiquiatr. clín.. 2007; 34 Suppl 2:169-73.

6. Del Cura MLA. Satisfação profissional do enfermeiro [dissertation]. Ribeirão Preto: Escola de Enfermagem de Ribeirão Preto/USP; 1994.

7. Bianchi ERF. Stress entre enfermeiros hospitalares. [livre docência]. São Paulo: Escola de Enfermagem de São Paulo/USP; 1999.

8. Guimarães J. Janelas do Santa Tereza: estudo do processo de reabilitação psicossocial do Hospital Psiquiátrico de Ribeirão Preto (SP). História, Ciência, Saúde- Manguinhos. 2001;8(2):357-74.

9. Furegato ARF. Relações interpessoais terapêuticas na enfermagem. Ribeirão Preto: Scala; 1999.

10. Furegato ARF, Saeki T. Formando especialistas em enfermagem psiquiátrica e saúde mental por mais de duas décadas. Cadernos IPUB. 2000; 6(19): 128-141.

11. Day JC, Bental RP, Roberto C, Randall F, Rogers A, Cattell D, et al. Attitudes toward a antipsychotic medication: the impact of clinical variables and relationships with health professionals. Arch Gen Psychiatry. 2005; 62(7): 717-24.

12. Costa VT, Lunardi VL, Lunard Filho WD. Autonomia versus cronicidade: uma questão ética no processo de cuidar em enfermagem. Rev. enferm. UERJ . 2007; 15(1):53-8.

13. Adams J, Scoott J. Predicting medication adherence in mental disorders. Acta Psychiatr Scand. 2000; 101(2): 119-24.

14. Keller MB, Hirschfeld RMA, Demyttenaere K, Baldwim DS. Optimizing outcomes in depression: focus on antidepressant compliance. Int Clin Psychopharmacol. 2002; 17(6): 265-71.
15. Silveira LMC, Ribeiro VMB. Grupo de adesão ao tratamento: espaço de "ensinagem" para profissionais de saúde e pacientes. Interface. 2008; 9(16): 91-104.

16. Poster EC, Ryan J. A multiregional study of nurse's beliefs and attitudes about work safety and patient assault. Hospital and Community Psychiatric Journal. 1994;45(11): 1104-8.

17. Teixeira MB, Barros S. Assistência de enfermagem a pacientes com manifestação de comportamento decorrente de alheamento da realidade. Rev. esc. enferm. USP. 1991; 25(3): 335-46.

18. Mello R, Furegato ARF. Internações psiquiátricas no Rio de Janeiro de 1996 a 2005. Rev. enferm. UERJ . 2007;15(2): 176-182.

19. Villares CC, Sartorius N. Challenginging the stigma of schizophrenia. Rev. Bras. Psiquiatr. 2003; 25(1): 1-2.

20. Andreasen NC, Carpenter WT, Kane JM, Lasser RA, Marder SR, Weinberger DR. Remission in Schizophrenia: proposed criteria and rationale for consensus. American Journal of Psychiatry. 2005; 162(3): 441-49.

Artigo recebido em 25.05.07.

Aprovado para publicação em 31.12.08. 